\title{
Human IgG Fc domain engineering enhances antitoxin neutralizing antibody activity
}

\author{
Stylianos Bournazos, ${ }^{1}$ Siu-Kei Chow, ${ }^{2}$ Nareen Abboud, ${ }^{2}$ \\ Arturo Casadevall,2,3 and Jeffrey V. Ravetch ${ }^{1}$
}

\begin{abstract}
1 Laboratory of Molecular Genetics and Immunology, The Rockefeller University, New York, New York, USA.
2Department of Microbiology and Immunology and ${ }^{3}$ Department of Medicine, Division of Infectious Diseases, Albert Einstein College of Medicine, New York, New York, USA.
\end{abstract}

\begin{abstract}
The effector activity of antibodies is dependent on engagement with $\mathrm{Fc} \gamma$ receptors (Fc $\gamma \mathrm{Rs}$ ) and activation of the associated intracellular signaling pathways. Preclinical evaluation of therapeutic humanized or chimeric $\mathrm{mAbs}$ to study the interactions of their Fc regions with $F c \gamma R s$ is hampered by substantial structural and functional $\mathrm{F} c \gamma \mathrm{R}$ diversity among species. In this report, we used mice expressing only human $\mathrm{F} c \gamma \mathrm{Rs}$ to evaluate the contribution of Fc $\gamma \mathrm{R}$-mediated pathways to the neutralizing activity of an anti-anthrax toxin chimeric $\mathrm{mAb}$. We observed that the protective activity of this $\mathrm{mAb}$ was highly dependent upon $\mathrm{Fc} \gamma \mathrm{R}$ engagement, with minimal protection against anthrax toxin observed in $\mathrm{Fc} \gamma \mathrm{R}$-deficient mice following $\mathrm{mAb}$ administration. We generated anti-anthrax toxin $\mathrm{mAbs}$ with specific $\mathrm{Fc}$ domain variants with selectively enhanced affinity for particular human Fc $\gamma$ Rs and assessed their activity in Fc $\gamma \mathrm{R}$-humanized mice. We determined that $\mathrm{Fc}$ domain variants that were capable of selectively engaging activating $F c \gamma R s$ substantially enhanced the in vitro and in vivo activity of anthrax toxin-neutralizing antibodies. These findings indicate that the application of Fc domain engineering is a feasible strategy to enhance toxin-neutralizing activity and suggest that engineered antitoxin antibodies will have improved therapeutic efficacy.
\end{abstract}

\section{Introduction}

The development of hybridoma technology has revolutionized medicinal therapeutics, making possible the generation of highly specific $m A b s$ with efficacy against a wide range of diseases. While Fab-antigen interactions play a crucial role in the protective activity of an antibody, it is now apparent that coupling the Fab-mediated recognition with Fc effector activity is crucial for optimal in vivo activity for protection against microbial pathogens and their toxins (1-5). Fcy receptors (FcyRs) are capable of either cellular activation through immunoreceptor tyrosine-based activation motif-dependent activation of intracellular tyrosine kinases or the inhibition of activation through recruitment of phosphatases to the immunoreceptor tyrosine-based inhibition motif domain and are thereby categorized into 2 broad classes: activating and inhibitory (6). The cellular outcome of IgG interaction with FcyRs is governed by the affinity of the $\mathrm{Fc}$ domain for the specific receptor and the expression pattern of those receptors on the effector cells. Since most effector cells coexpress activating and inhibitory Fc $\gamma$ Rs, it is the ratio of the binding affinities of a specific IgG Fc to these receptors that will determine the outcome of the IgG-Fc $\gamma \mathrm{R}$ interaction (7). Indeed, differences in the capacity of an IgG molecule to engage activating or inhibitory $\mathrm{F} c \gamma \mathrm{Rs}$ are a determining factor for the in vivo activity of a particular IgG subclass or variant $(3,7)$.

Antibody-mediated neutralization of bacterial toxins was classically considered to be a direct process that relied solely on the ability of the variable region of antibodies to bind toxins. However, recent findings suggest that effective in vivo protection against microbial pathogens and their toxins requires both Fab recognition and Fc binding to FcyRs for optimal activity $(1,8-10)$. This

Authorship note: Arturo Casadevall and Jeffrey V. Ravetch are co-senior authors. Conflict of interest: The authors have declared that no conflict of interest exists. Citation for this article: J Clin Invest. 2014;124(2):725-729. doi:10.1172/JCI72676. in turn suggests that it may be possible to enhance the toxin-neutralizing activity of antibodies by engineering the $\mathrm{Fc}$ domain to selectively engage certain classes of Fc $\gamma$ Rs. Indeed, engineering of the $\mathrm{Fc}$ region of an immunoglobulin can increase its protective efficacy against different pathogens and improve effector functions, including antibody-dependent cell-mediated cytotoxicity and opsonization (11-13).

Over the past decade, significant advances have been made to generate humanized and mouse-human chimeric mAbs to reduce toxicity and enhance various effector functions (14). Murine or nonhuman primate model systems are commonly used for the preclinical evaluation of these $\mathrm{mAbs}$ and for the study of the Fc-FcyR interaction for humanized/chimeric antibodies, even though these models poorly reflect the structural diversity and the unique expression pattern of human FcyRs of human leukocytes (15-18). Therefore, we have recently developed a mouse model in which the mouse FcyRs were deleted and all the human FcyRs were expressed as transgenes, recapitulating the human-specific expression pattern (19). Since these mice retain functional $\mathrm{F} \gamma \gamma \mathrm{R}$ binding and signaling activities, their use facilitates the assessment of the neutralization activity of human mAbs in a context closely related to the human FcyR system. Here, we used the well-characterized anthrax toxin neutralization model $(1,10)$ to study the role of Fc $\gamma \mathrm{R}$-mediated pathways in its neutralization activity using Fc $\gamma$ R-humanized mice and mouse-human chimeric forms of a protective mAb. Additionally, we report that specific $\mathrm{Fc}$ domain variants of this $\mathrm{mAb}$ present significantly augmented in vitro and in vivo neutralization activity through selective engagement of particular classes of human FcyRs.

\section{Results and Discussion}

Toxin neutralization was previously thought to be the sole result of blocking toxin activity through Fab-antigen interactions. However, our recent observations indicate that FcyR-mediated path- 
A

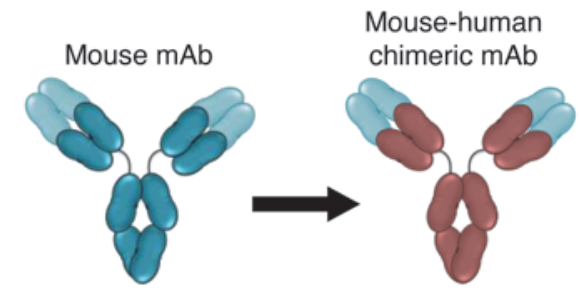

B
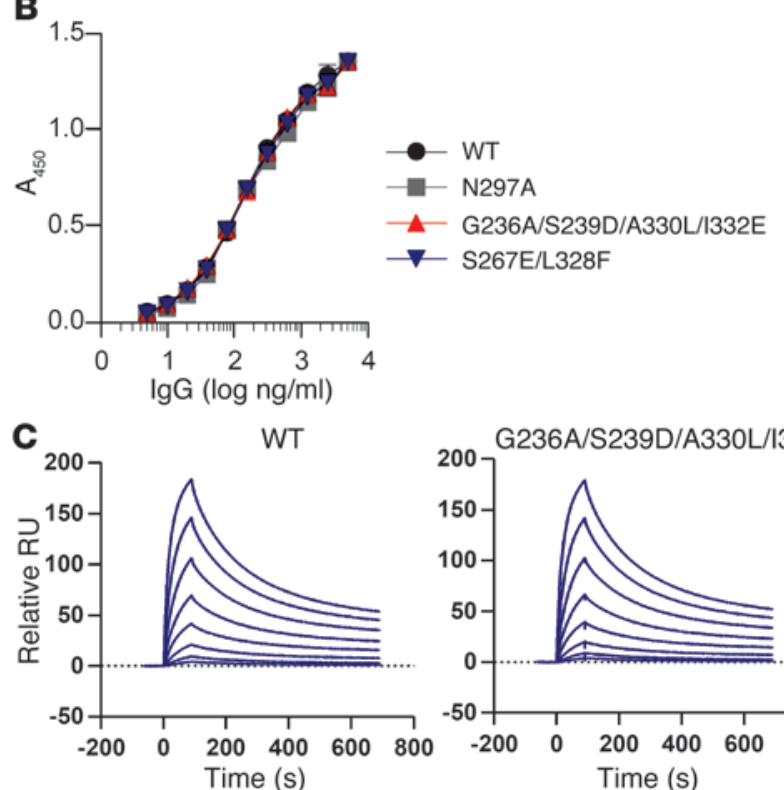

G236A/S239D/A330L/I332E

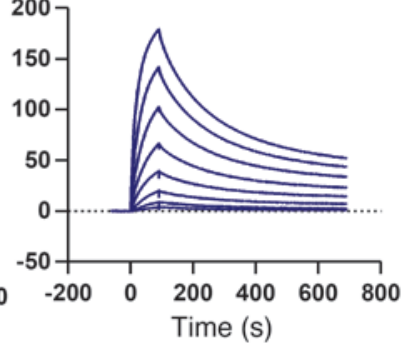

ways, such as Fc-mediated toxin uptake by effector cells like macrophages, contribute substantially to the neutralizing activity of anti-anthrax toxin mAbs $(1,10)$. Given the previously described requirement for $\mathrm{Fc} \gamma \mathrm{Rs}$ for the activity of anti-anthrax mouse $\mathrm{mAb}$ 19D9 (1), we have generated a mouse-human chimeric IgG form of this antibody as a necessary precursor to a fully human therapeutic antibody. The constant regions of the heavy and light chains (mouse IgG1,א) were replaced with those of human IgG1 (hIgG1) and $\kappa$ constant regions, respectively (Figure $1 \mathrm{~A}$ ).

Similarly, we have shown previously that generation of switch variants of the parental 19D9 mAb and comparison of their neutralization potencies resulted in more effective neutralizing antibodies, while not effecting their binding affinity or specificity, such that their relative efficacy was IgG2a>IgG2b>IgG1 (1). This hierarchy of mouse IgG subclass activity results from the differential binding of these subclasses to specific mouse FcyRs, as previously determined (7). Mouse IgG2a binds with a log higher affinity to the activation receptor Fc $\gamma$ RIV, while mouse IgG1 preferentially engages the inhibitory $\mathrm{Fc} \gamma \mathrm{R}, \mathrm{Fc} \gamma \mathrm{RIIb}$ (7). These differences in the capacity of mouse IgG subclasses to engage activating or inhibitory Fc $\gamma$ Rs was previously shown to be predictive of the in vivo antibody-dependent cell-mediated cytotoxicity activity in several mouse models $(7,19)$. In contrast to the murine IgG subclasses, no specific human IgG subclass exists that binds selectively to a particular class of human Fc $\gamma$ Rs conferring enhanced in vivo activity, such as mouse IgG2a, which interacts selectively with activating Fc $\gamma$ Rs (7).

Since the main determining factor for the $\mathrm{Fc}-\mathrm{Fc} \gamma \mathrm{R}$ interactions is the amino acid backbone of the Fc region, we generated specific mutants of hIgG1 19D9 within the Fc domain region that par-

\section{Figure 1}

Generation and characterization of mouse-human chimeric anti-PA mAb. (A) Representation of the mouse-human chimeric anti-PA (19D9) mAb. The constant regions of the heavy ( $\mathrm{CH} 1-3)$ and light chain (СК) of mouse IgG1, $\kappa$ (parental mAb) were replaced with those of hlgG1 and $\mathrm{C}_{\kappa}$, respectively. Recombinant 19D9 hlgG1 wild-type and Fc domain variants were generated. No difference in their affinity and specificity for PA was evident among the various Fc domain variants, as assessed by (B) ELISA and (C) surface plasmon resonance (affinity values are shown in Supplemental Table 2).

ticipates in the $\mathrm{Fc}-\mathrm{F} c \gamma \mathrm{R}$ interface. We characterized the effect of different mutations on the affinity for the different human $F c \gamma R$ classes and identified variants that exhibit differential binding affinities for the various human FcyR classes. These Fc domain variants had the capacity for enhanced engagement of either activating Fc $\gamma$ Rs, such as Fc $\gamma$ RIIa and Fc $\gamma$ RIIIa (G236A/S239D/ A330L/I332E), or the inhibitory Fc $\gamma$ RIIb (S267E/L328F) (Supplemental Table 1; supplemental material available online with this article; doi:10.1172/JCI72676DS1). Additionally, an Fc domain variant exhibiting minimal binding (N297A) for all classes of human Fc $\gamma$ Rs was generated (Supplemental Table 1).

Wild-type hIgG1 and Fc domain variants of 19D9 were expressed in mammalian cells, and their specificity and affinity to the antigen, protective antigen (PA) of Bacillus anthracis, were determined by ELISA (Figure 1B) and surface plasmon resonance (Figure 1C and Supplemental Table 2). While these $\mathrm{Fc}_{\mathrm{c}}$ domain variants exhibited differential binding capacity for human Fc $\gamma$ Rs, no differences in their affinity and specificity for PA were evident. This observation is consistent with a prior observation that isotype switching of the mouse IgG1-producing hybridoma 19D9 to produce mIgG2a and mIgG2b had no effect on specificity, implying that the 19D9 $\mathrm{V}$ regions are not susceptible to the Fc-mediated effects on affinity that have been described for other mAbs (20).

The protective efficacy of mouse-human chimeric 19D9 mAb was assessed using mice expressing all classes of human FcyRs

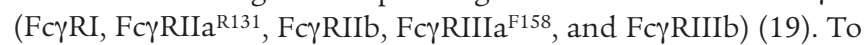
determine the contribution of $\mathrm{Fc} \gamma \mathrm{R}$-mediated pathways to the activity of the chimeric 19D9 hIgG1 mAb, bone marrow-derived macrophages (BMDMs), the main target of the anthrax lethal toxin (LeTx) during anthrax infection, were obtained from mice lacking all genes encoding for $F c \gamma$ Rs $\left(F c \gamma R \alpha^{\text {null }}\right)$ and from $F c \gamma R \alpha^{\text {null }}$ mice expressing human Fc $\gamma$ Rs (Fc $\gamma$ R-humanized mice). Comparison of the in vitro neutralization activity of the chimeric hIgG1 19D9 $\mathrm{mAb}$ in $\mathrm{Fc} \gamma \mathrm{R} \alpha^{\text {null }}$ and $\mathrm{Fc} \gamma \mathrm{R}$-humanized cells revealed significantly decreased protective activity in the absence of $\mathrm{Fc} \gamma \mathrm{Rs}$, as evidenced by enhanced anthrax LeTx-mediated cytotoxicity in Fc $\gamma R \alpha^{\text {null }}$ BMDMs compared with that in FcyR-humanized BMDMs (Figure 2A) (hIgG1 19D9 IC $_{50}$ in FcyR-humanized BMDMs, $0.21 \mu \mathrm{g} \mathrm{ml}^{-1}$; hIgG1 19D9 $\mathrm{IC}_{50}$ in Fc $\gamma \mathrm{R}^{\mathrm{null}}, 21.45 \mu \mathrm{g} \mathrm{ml}^{-1}$ ). Analysis of the expression of human Fc $\gamma$ Rs of BMDMs obtained from Fc $\gamma$ R-humanized mice showed an expression pattern similar to that of primary human macrophages (18). In particular, Fc $\gamma$ R-humanized BMDMs expressed Fc $\gamma$ RIIa (CD32a), Fc $\gamma$ RIIb (CD32b), and Fc $\gamma$ RIIIa (CD16a), while they lacked Fc $\gamma \mathrm{RI}$ (CD64) and $\mathrm{F} c \gamma \mathrm{RIIIb}$ (CD16b) (Figure 2B). We next determined the protective activity of the mouse-human chimeric hIgG1 19D9 mAb in vivo using $\mathrm{Fc} \gamma \mathrm{R}$-humanized mice and $\mathrm{Fc} \gamma \mathrm{R} \alpha^{\text {null }}$ mice. While the hIgG1 19D9 mAb (750 $\mu$ g; i.p. 3 hours prior to bacterial challenge) was protective in $\mathrm{F} c \gamma \mathrm{R}$-humanized mice following the i.v. challenge 


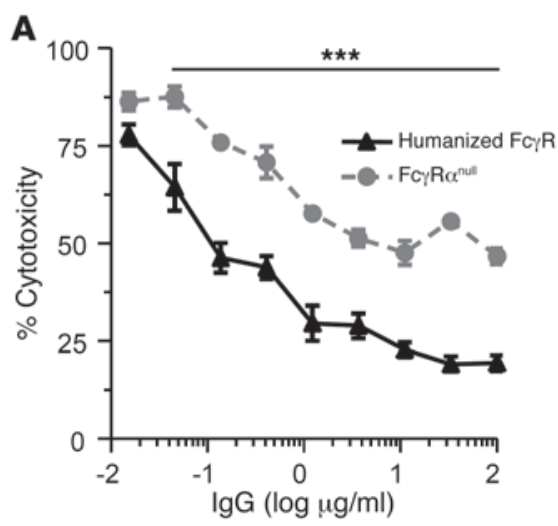

B
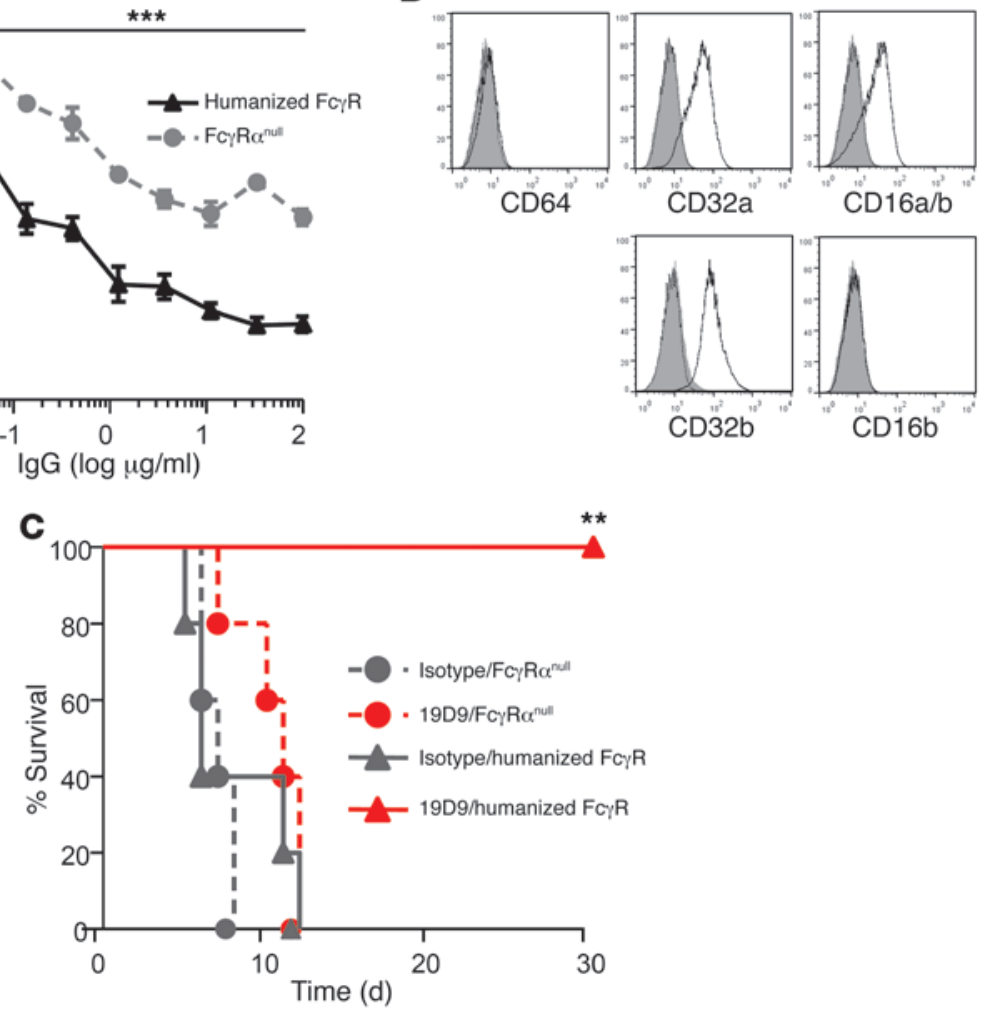

\section{Figure 2}

$\mathrm{Fc} \gamma \mathrm{R}$ requirement for the neutralizing activity of the anti-PA mouse-human chimeric mAb. (A) The protective activity of 19D9 hlgG1 against anthrax LeTx was compared in BMDMs obtained either from humanized Fc $\gamma \mathrm{R}$ (wild-type) or Fc $\gamma \mathrm{R}$-deficient (FcyR $\left.\alpha^{\text {null }}\right)$ mice. $n=4 ;{ }^{* \star *} P<0.0001 ; n=4$. (B) Human Fc $\gamma \mathrm{R}$ expression profile of BMDMs from humanized Fc $\mathrm{R}$ (solid black line) or $F_{c} \gamma R \alpha^{\text {null }}$ (solid gray filled) mice (isotype control; dotted line). (C) Comparison of the protective activity of 19D9 hlgG1 $(750 \mu \mathrm{g}$ i.p.) in humanized $F_{C} \gamma R$ and $F_{C} \gamma R_{\alpha}{ }^{\text {null }}$ mice following challenge with $B$. anthracis Sterne strain. $n=5$ per group; ${ }^{\star \star} P=0.005, F c \gamma R \alpha^{\text {null }}$ vs. humanized Fc $\gamma$ R. with $B$. anthracis Sterne strain $\left(10^{4}\right.$ cells), no activity was observed in Fc $\gamma$ R-deficient mice (Figure 2C). These findings clearly suggest the requirement of $\mathrm{Fc} \gamma \mathrm{R}$ engagement in the protective activity of hIgG1 19D9 against anthrax toxin.

Since the protective activity of the mAb 19D9 to LeTx is dependent upon $\mathrm{F} c \gamma \mathrm{R}$ engagement, we hypothesized that alterations in its capacity to engage particular classes of Fc $\gamma$ Rs could modulate its neutralizing potency. To test this concept, the in vivo neutralizing activity of different Fc domain variants of hIgG1 19D9 was compared using Fc $\gamma$ R-humanized mice. These variants have been characterized in terms of Fc $\mathrm{R}$ binding, and they presented enhanced affinity for either activating or inhibitory human Fc $\gamma$ Rs (Supplemental Table 1). When compared with wild-type hIgG1 19D9, the G236A/S239D/A330L/I332E variant, which has increased affinity for activating Fc $\gamma$ Rs, including Fc $\gamma$ RIIa (13-fold increase) and Fc $\gamma$ RIIIa (15-fold increase), demonstrated substantially augmented protective activity in vitro (10-fold improved potency - wild-type hIgG1 IC $\mathrm{I}_{50}, 0.31 \mathrm{\mu g} \mathrm{ml}^{-1}$; G236A/S239D/ A330L/I332E IC $50,0.03 \mu \mathrm{g} \mathrm{ml}^{-1}$ ).

To assess whether the G236A/S239D/A330L/I332E variant also displayed augmented activity in vivo, we selected a suboptimal dose $(350 \mu \mathrm{g})$, at which the wild-type hIgG1 19D9 was ineffective (Figure $3 \mathrm{~B}$ ). As is evident from Figure 3B, >70\% survival was achieved with $350 \mu \mathrm{g}$ G236A/S239D/A330L/I332E 19D9, while no significant protective activity was observed with wild-type hIgG1 $19 \mathrm{D} 9$ at this particular dose, suggesting that administration of the G236A/S239D/A330L/I332E variant lowered the threshold of $\mathrm{mAb}$ required to fully confer in vivo protection.

In contrast, no major difference was observed when we compared the activity of wild-type IgG1 19D9 with that of the S267E/ L328F variant, which binds with enhanced affinity to Fc $\gamma$ RIIa and the inhibitory Fc $\gamma$ RIIb (44-fold and 113-fold increase for Fc $\gamma$ RIIa and FcyRIIb, respectively; Supplemental Table 1) (Figure 3, A and C). Minimal in vitro and in vivo neutralizing activity was observed for the Fc $\gamma$ R null binding mutant (N297A), further supporting the requirement for $\mathrm{F} c \gamma \mathrm{R}$ for the $\mathrm{mAb}$-mediated protection against B. anthracis infection (Figure 3, A and C).

These findings suggest that preferential engagement of activating FcyRs does enhance the neutralization activity of the 19D9 $\mathrm{mAb}$ in humans and are consistent with our previous observations in wild-type mice, in which the mouse IgG2a 19D9 isotype variant was more effective compared with mouse IgG1 (1). In contrast, minimal contribution of the inhibitory FcyRIIb to the mAb-mediated toxin neutralization was evident, as the FcyRIIb-enhanced binding Fc domain variant of 19D9 (S267E/ L328F) displayed activity comparable to that of wild-type hIgG1. Although this variant (S267E/L328F) also displayed enhanced binding to the activating Fc $\gamma$ RIIa, its substantially augmented binding capacity for FcyRIIb is likely to overcome any cellular activation signals following Fc $\gamma$ RIIa coengagement, especially given the higher expression levels of Fcy RIIb compared with Fc $\gamma$ RIIa in all leukocyte types $(18,19)$. This assumption is further strengthened by our previous observations from mAb-mediated cellular cytotoxicity models in Fc $\gamma$ R-humanized mice, in which the S267E/L328F variant failed to display enhanced in vivo cytotoxic activity, despite its capacity to engage FcrRIIa (19).

As we have previously demonstrated (1), mouse 19D9 mAb (irrespective of IgG subclass) failed to protect against B. anthracis infection in mice deficient in activating $\mathrm{Fc} \gamma \mathrm{Rs}(\mathrm{FcR} \gamma$-chain knockout $\left[\mathrm{Fcer}_{1 \mathrm{~g}^{-/}}\right]$mice) or in all classes of Fc $\gamma \mathrm{Rs}\left(\mathrm{Fcer}_{\mathrm{g}} \mathrm{g}^{--}\right.$and $F_{c g r} 2 b^{-/-}$mice), further excluding a role of Fc $\gamma$ RIIb in the in vivo neutralizing activity. It is likely that increased $\mathrm{mAb}$ interactions 


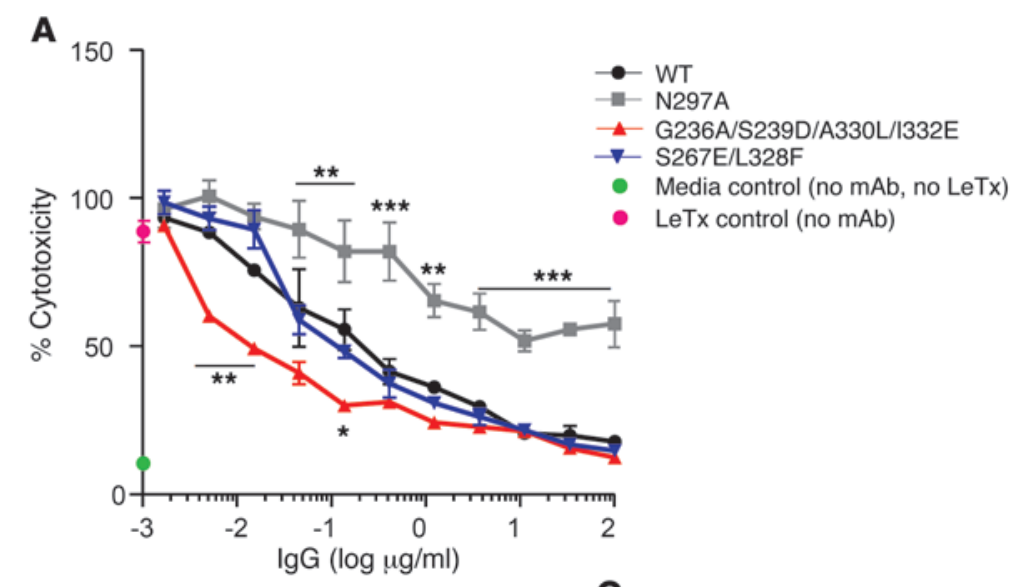

B

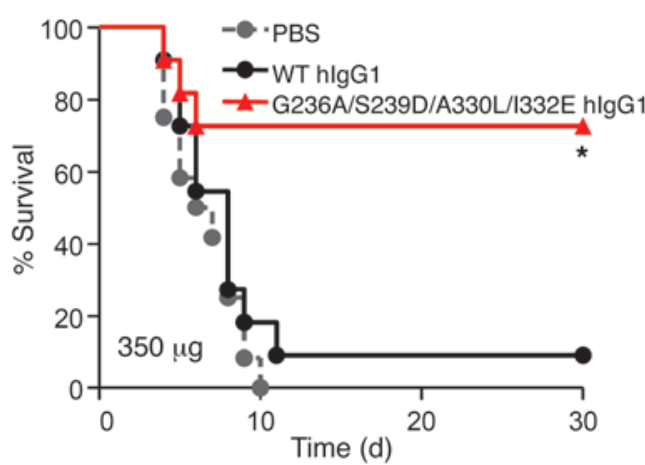

C

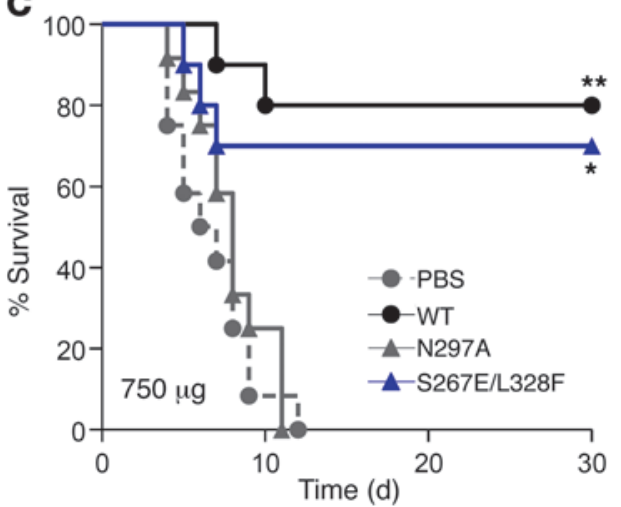

Figure 3

Enhancement of the neutralization activity of anti-PA hlgG1 mAb through Fc domain engineering. Fc domain variants of $19 \mathrm{D} 9 \mathrm{hlgG} 1$ with differential binding capacity for the various classes of human $\mathrm{Fc} \gamma \mathrm{Rs}$ were generated and their neutralization activity was assessed both (A) in vitro and (B and $\mathbf{C}$ ) in vivo. (A) LeTx-induced cytotoxicity was assessed in BMDMs in the presence of the different Fc domain variants of 19D9 hlgG1. $n=2 ;{ }^{*} P<0.05,{ }^{* *} P<0.01,{ }^{* *} P<0.001$, vs. wild-type IgG1. (B) Enhanced in vivo protective activity of the G236A/S239D/A330L/I332E 19D9 hlgG1 variant in FcyR-humanized mice challenged with $B$. anthracis. Mice received the indicated mAb variant (350 $\mu \mathrm{g})$ or PBS i.p. 3 hours prior to challenge. $n=11-12$ per group; ${ }^{*} P=0.0094$, compared with wild-type hlgG1 group. (C) The neutralization activity of 19D9 hlgG1 Fc domain variants $(750 \mu \mathrm{g}$ i.p.) with differential $\mathrm{Fc} \gamma \mathrm{R}$ binding capacity was compared in humanized $\mathrm{Fc} \gamma \mathrm{R}$ mice following challenge with $B$. anthracis. $n=10-12$ per group; ${ }^{*} P=0.005,{ }^{* *} P=0.002$, compared with N297A group.

with activating FcyRs lead to substantially enhanced protection during $B$. anthracis infection through modulation of the phagocytic activity of FcyR-expressing effector cells, augmenting the clearance of PA-anti-PA immune complexes and preventing the formation of anthrax LeTx. Additionally, since the G236A/ S239D/A330L/I332E IgG1 Fc variant (and particularly the A330L mutation; ref. 21) is deficient in mouse and human C1q binding and complement activation, a role for complementmediated pathways in the in vivo $\mathrm{mAb}$-mediated neutralization activity of 19D9 could be excluded.

In summary, we have shown that the toxin neutralization activity of a mouse-human antibody against the anthrax LeTx is dependent on interactions with human FcyRs and identified an optimized human $\mathrm{F}_{\mathrm{C} \gamma \mathrm{R}}$ binding profile for toxin-neutralizing efficacy. In the past, the evaluation of the in vivo activity in wild-type mice of human mAbs intended for human therapeutics, like 19D9, was hampered by the substantial interspecies differences both in structure and in the function of $\mathrm{Fc \gamma}$ Rs as well as their expression pattern. In this study, we have circumvented that prior limitation by using FcyR-humanized mice, which faithfully recapitulate the unique pattern of human $\mathrm{F} \gamma \gamma \mathrm{R}$ expression observed in the various human tissues. So, our findings, obtained with the use of FcyR-humanized mice, are physiologically relevant to the context of human B. anthracis infection. Additionally, we showed that the in vivo neutralization activity of mouse-human chimeric 19D9 mAb offers significant advantages over conventional strategies using wild-type mice, thus allowing the precise characterization of the activity of Fc domain variants with altered capacity to engage specific classes of human FcyRs. Most importantly, our findings establish that it is possible to enhance the toxin-neutralizing activity of an antibody by manipulating $\mathrm{Fc}$-mediated interactions. Indeed, increased endocytic uptake of mAb-opsonized PA by augmented engagement of activating FcrRs on effector cells could prevent furin-mediated cleavage of PA and the subsequent assembly of the anthrax LeTx $(1,10)$. That finding in turn suggests strategies for designing more effective therapeutic antibodies for toxin neutralization and vaccines that elicit superior toxin-neutralizing responses through the production of antibodies that engage activating $\mathrm{F} c \gamma \mathrm{Rs}$.

\section{Methods}

A detailed description of the methods is provided in the Supplemental Methods. 
Mice and in vivo protection experiments. Mice deficient for all classes of murine $\mathrm{Fc} \gamma \mathrm{Rs}\left(\mathrm{F} c \gamma \mathrm{R} \alpha^{\text {null }}\right.$ mice) and $\mathrm{F} c \gamma \mathrm{R}$-humanized mice have been previously characterized (19). For in vivo protection experiments, 19D9 or isotype antibody was administered i.p. 3 hours prior to i.v. challenge with $B$. anthracis Sterne strain $\left(10^{4}\right.$ cells).

Statistics. Quantitative data from multiple experiments are presented as mean \pm SEM. Two-way ANOVA with Bonferroni post-hoc $t$ test ( 2 tailed) was used to test for differences in in vitro protection. For in vivo protection experiments, survival rates were analyzed with the log-rank test. $P$ values of less than 0.05 were considered to be statistically significant.

Study approval. All in vivo experiments were performed in compliance with US federal laws and institutional guidelines of The Rockefeller University and have been approved by The Rockefeller University Institutional Animal Care and Use Committee.

1. Abboud $\mathrm{N}$, et al. A requirement for $\mathrm{F} c \gamma \mathrm{R}$ in antibody-mediated bacterial toxin neutralization. J Exp Med. 2010;207(11):2395-2405.

2. Anthony RM, Wermeling F, Ravetch JV. Novel roles for the IgG Fc glycan. Ann N Y Acad Sci. 2012; 1253:170-180.

3. Li F, Ravetch JV. Inhibitory Fcr receptor engagement drives adjuvant and anti-tumor activities of agonistic CD40 antibodies. Science. 2011;333(6045):1030-1034.

4. Corti D, et al. A neutralizing antibody selected from plasma cells that binds to group 1 and group 2 influenza A hemagglutinins. Science. 2011; 333(6044):850-856.

5. Hessell AJ, et al. Fc receptor but not complement binding is important in antibody protection against HIV. Nature. 2007;449(7158):101-104.

6. Nimmerjahn F, Ravetch JV. Fcy receptors as regulators of immune responses. Nat Rev Immunol. 2008; 8(1):34-47.

7. Nimmerjahn F, Ravetch JV. Divergent immunoglobulin g subclass activity through selective Fc receptor binding. Science. 2005;310(5753):1510-1512.

8. Ngundi MM, Meade BD, Lin TL, Tang WJ, Burns DL. Comparison of three anthrax toxin neutralization assays. Clin Vaccine Immunol. 2010;17(6):895-903.

\section{Acknowledgments}

The authors wish to thank Johanna Rivera and all the members of the Laboratory of Molecular Genetics and Immunology for the helpful discussions and support. This work was supported by grants from the NIH and the Northeast Biodefense Center (NBC) (to J.V. Ravetch). S. Bournazos was supported by an NBC Career Development Award (5U54AI057158).

Received for publication August 12, 2013, and accepted in revised form October 30, 2013.

Address correspondence to: Jeffrey V. Ravetch, 1230 York Ave., Box 98, New York, New York 10065, USA. Phone: 212.327.7321; Fax: 212.327.7319; E-mail: ravetch@rockefeller.edu.

9. Akiyoshi DE, Sheoran AS, Rich CM, Richard L, Chapman-Bonofiglio S, Tzipori S. Evaluation of Fab and $F\left(a b^{\prime}\right) 2$ fragments and isotype variants of a recombinant human monoclonal antibody against Shiga toxin 2. Infect Immun. 2010;78(3):1376-1382.

10. Chow SK, Smith C, MacCarthy T, Pohl MA, Bergman A, Casadevall A. Disease-enhancing antibodies improve the efficacy of bacterial toxin-neutralizing antibodies. Cell Host Microbe. 2013;13(4):417-428.

11. Lazar GA, et al. Engineered antibody Fc variants with enhanced effector function. Proc Natl Acad Sci U S A. 2006;103(11):4005-4010.

12. Heider $\mathrm{KH}$, et al. A novel Fc-engineered monoclonal antibody to CD37 with enhanced ADCC and high proapoptotic activity for treatment of B-cell malignancies. Blood. 2011;118(15):4159-4168.

13. Horton HM, et al. Fc-engineered anti-CD40 antibody enhances multiple effector functions and exhibits potent in vitro and in vivo antitumor activity against hematologic malignancies. Blood. 2010; 116(16):3004-3012.

14. Casadevall A, Dadachova E, Pirofski LA. Passive antibody therapy for infectious diseases. Nat Rev Microbiol. 2004;2(9):695-703.

15. Oliphant T, et al. Development of a humanized monoclonal antibody with therapeutic potential against West Nile virus. Nat Med. 2005;11(5):522-530.

16. Prigent $J$, et al. Production and characterisation of a neutralising chimeric antibody against botulinum neurotoxin A. PLoS One. 2010;5(10):e13245.

17. Mohamed $\mathrm{N}$, et al. A high-affinity monoclonal antibody to anthrax protective antigen passively protects rabbits before and after aerosolized Bacillus anthracis spore challenge. Infect Immun. 2005; 73(2):795-802.

18. Bournazos S, Woof JM, Hart SP, Dransfield I. Functional and clinical consequences of $\mathrm{Fc}$ receptor polymorphic and copy number variants. Clin Exp Immunol. 2009;157(2):244-254.

19. Smith P, DiLillo DJ, Bournazos S, Li F, Ravetch JV. Mouse model recapitulating human Fcgamma receptor structural and functional diversity. Proc Natl Acad Sci U S A. 2012;109(16):6181-6186.

20. Casadevall A, Janda A. Immunoglobulin isotype influences affinity and specificity. Proc Natl Acad Sci U S A. 2012;109(31):12272-12273.

21. Mehlhop E, et al. Complement protein $\mathrm{C} 1 \mathrm{q}$ reduces the stoichiometric threshold for antibody-mediated neutralization of West Nile virus. Cell Host Microbe. 2009;6(4):381-391. 